\title{
Smart Sensor Network Based High Quality Air Pollution Monitoring System Using Labview
}

\author{
https://doi.org/10.3991/ijoe.v13i08.7161 \\ Nagarjuna Telagam( $\left.{ }^{\varpi}\right)$, Nehru Kandasamy, Nagendra Prasad G \\ Institute of Aeronautical Engineering, Hyderabad, India \\ nagarjuna473@gmail.com \\ Menakadevi Nanjundan \\ Hindustan College of Engineering and Technology Coimbatore, India
}

\begin{abstract}
A ZigBee based wireless sensor network is implemented in this paper which is of low-cost solar-powered air quality monitoring system. The main objective of the proposed architecture is to interfacing various sensors to measure the sensor analog data and displayed in LabVIEW on the monitor using the graphical user interface (GUI). The real time ambient air quality monitoring in smart cities is of greater significance for the health of people. The wireless network sensor nodes are placed at different traffic signals in the smart cities which collect and report real-time data on different gases which are present in the environment such as carbon monoxide (CO), nitrogen dioxide ( $\left.\mathrm{NO}_{2}\right)$, methane $\left(\mathrm{CH}_{4}\right)$ and humidity. The proposed system allows smart cities to monitor air quality conditions on a desktop/laptop computer through an application designed using graphical programming based LabVIEW software and provides an alert if the air quality characteristics exceed acceptable levels. The sensor network was successfully tested on the campus of the institute of aeronautical engineering, Hyderabad. The sensor data are indicated by different indicators on the front panel of LabVIEW and also different charts are plotted with respect to time and amplitude which explains the severity of polluted areas.
\end{abstract}

Keywords-LabVIEW, Air Pollutants, wireless sensor network

\section{Introduction}

Over the few years, the increasing body of epidemiological has led to the harmful effects of ambient air pollution on health which leads to heart diseases. The several environmental air pollutants such as $\mathrm{CO}, \mathrm{NO}_{2}$ and particulate matter. These harmfulair pollutants are associated with increased integrity due to cardiovascular disease [1]. Especially Particulate matter 2.5 micrometres (PM2.5) is produced by incomplete combustion of gasoline, naphtha and with the help of steam biomass produces electricity This particulate matter can penetrate deep into the lungs and blood stream which causes diseases [2]. The world health organization (W.H.O) has mentioned that $93 \%$ of the population was living in places where the air quality guidelines 
levels were not met in 2014. The air pollutants cause 5 million premature deaths worldwide in 2014 [3]. Foremost studies have commonly depended on the traffic proximity to estimate subjection between air pollution and asthma incidence [4]. Traffic-related pollution exposure at different smart cities may contribute to the development of asthma, chronic obstructive pulmonary disease which is a lung disease, due to cigarette smoking the diseases such as emphysema and chronic bronchitis will occur [5]. If the diameter of particular matter is less than $2.5 \mu \mathrm{m}\left(\mathrm{PM}_{2.5}\right)$ and less than $10 \mu \mathrm{m}$ (PM10), can settle in the bronchi and lungs and cause health problems [6].Ambient air quality data from a central monitoring station were used to assign exposures to the pollutants [7]. Current screening tools and public guidance on school sitting are either too coarse in their spatial resolution for assessing individual sites [8]. The survey was conducted which includes 5,000 members in the age group of $20-30$, aimed at screening their lung health. The results indicated that most of the people are affected by lung disease and asthma because they have dehydrated with huge amounts of air pollutants.

The pollution in the smart cities is shown in Figure 1.Especially in between the age group between 8-14 years the worst affected are children they are more exposed to dust particles in the air [9]. The Southern California Children's Health Study (CHS) was launched in 1992 to evaluate the potential health effects in children of long -term exposures to poor air quality. CHS research results has shown different areas of ambient air pollution are observed between residential proximity to busy roads $(<75 \mathrm{~m})$ and asthma prevalence), as well as between residential proximity to freeways $(<500 \mathrm{~m})$ and both asthma and reduced rates of lung function growth However, the reported associations between residential proximity to busy roads and childhood asthma are inconsistent suggesting roadway proximity may not be a sufficiently adequate proxy for TRP exposure [10].

The architecture of the proposed air quality monitoring system is shown in Figure 2. Which consists of ZigBee end nodes, LCD, ATMEGA16, MQ-4, MQ-7, MQ-135 and a wireless gateway including associated software for system devices and a LabVIEW graphical user interface (GUI) for display of data on a remote computer/laptop. The end nodes (also known as sensor nodes) positioned at different poles in the traffic area scan simultaneously measure air quality parameters in an ambient environment. The coordinator is used to receive data transmitted from routers or end devices. The gateway provides data to a computer where the sensing data is stored and integrated into a database. The user can thus make inquiries for the history and latest data through the LabVIEW interface. By using different indicators in LabVIEW the output of analog data from the sensor is seen and plotted in various graphs and charts. 
Paper-Smart Sensor Network Based High Quality Air Pollution Monitoring System Using Labview

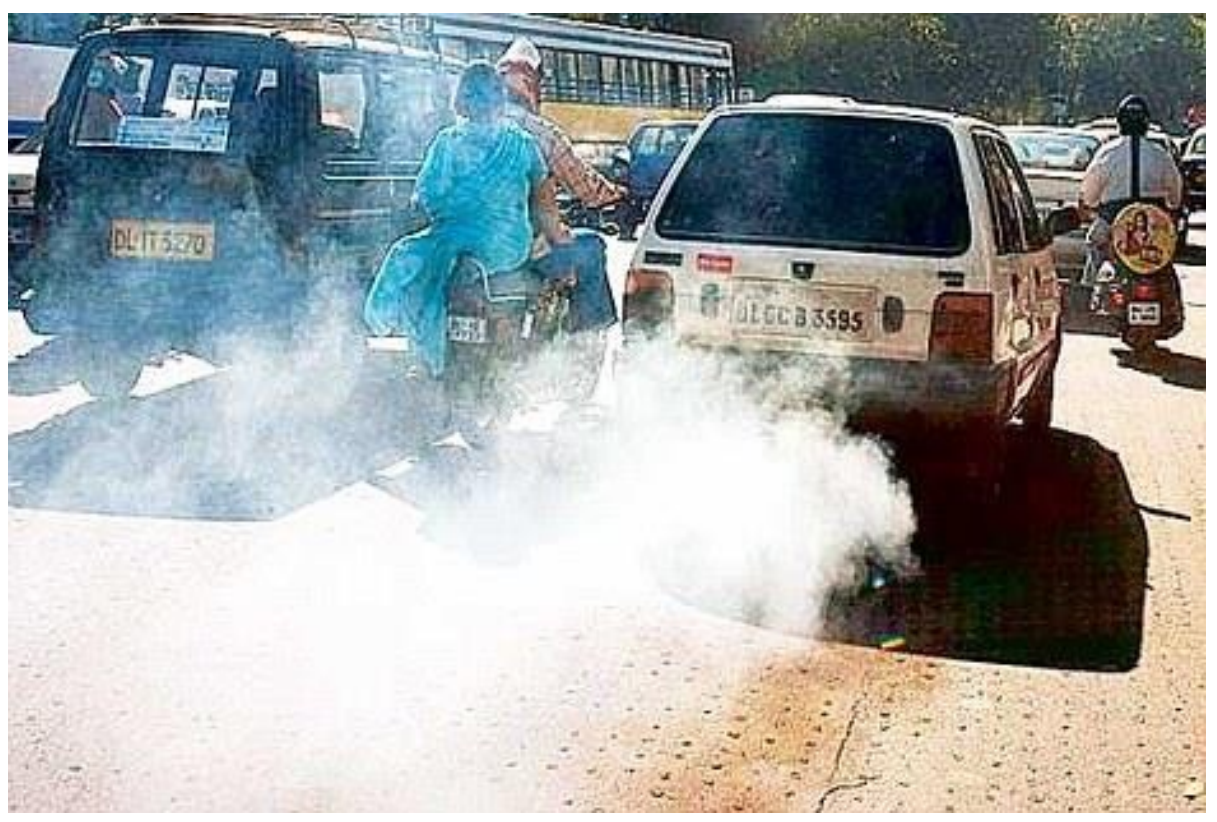

Fig. 1. Pollution in Hyderabad City [3]

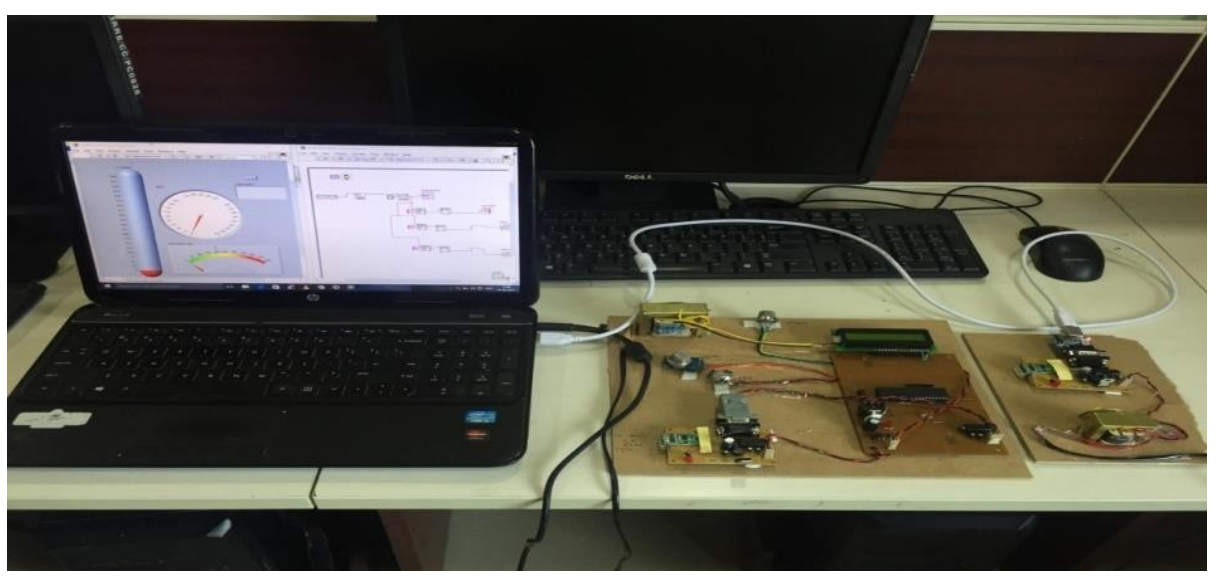

Fig. 2. WSN proposed Architecture 


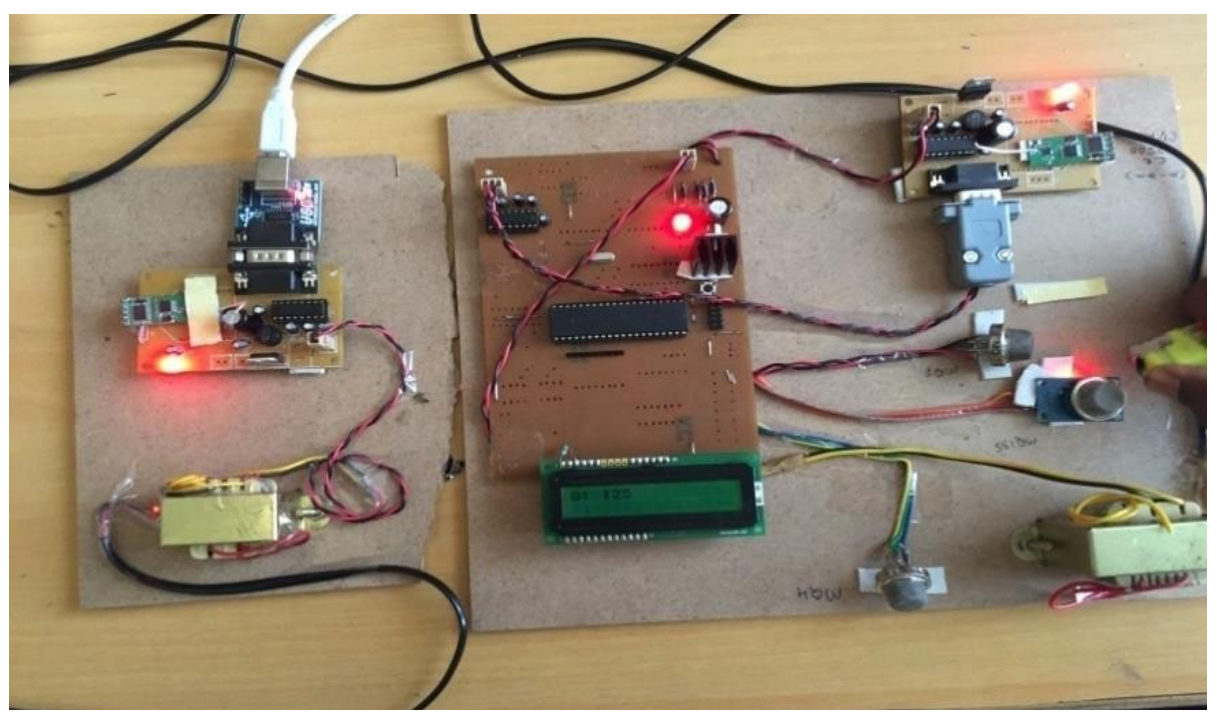

Fig. 3. Embedded system kit with three sensors

Embedded system kit with different air pollutants sensors are shown in Figure 3. Initially, the hardware kit is switched $\mathrm{ON}$, with the help of step down transformer, rectifier and voltage regulator $230 \mathrm{~V}$ AC is stepped down to $12 \mathrm{~V}$ DC. Here three different sensors such as MQ7, MQ4, MQ 135 are used to monitor the air pollution which captures the analog signal. The MQ-7 can detect CO-gas concentrations anywhere from 20 to $2000 \mathrm{ppm}$. The MQ-4 gas sensor has high sensitivity to Methane, also to Propane and Butane. The sensor could be used to detect different combustible gas, especially Methane. MQ135 gas sensor has high sensitivity to Ammonia, Sulfide and Benz E steam, also sensitive to smoke and other harmful gases Each sensor is connected to microcontroller which has four ports, port B is left alone. Zigbee module is used which is software based protocol and it is connected to the laptop through a serial cable. The VISA driver software is installed in laptop which supports serial communication. Liquid crystal displays a type of display used in digital watches and many portable computers. The data is given to the serial port which is given to read buffer, which specifies the data on the LCD display. For example, C: 234, N: $320, \mathrm{G}: 278$ is displayed on the LCD display and it is of 15 bytes length, the read buffer function is used on the back panel in the LabVIEW software to read the serial data.

A schematic, or schematic diagram, is a representation of the elements of a system using abstract, graphic symbols rather than realistic pictures. Here all the elements in the system are placed sequentially with resistors, capacitors and integrated circuits. Figure 4 shows the schematic diagram of proposed architecture.

The Here the while loop is taken with ' $i$ ' as the iteration parameter and for each iteration the delay time of $10 \mathrm{~ms}$ is considered. The output of input and output communication port is connected to VISA serial port and I/O COM1 acts as VISA resource name. Figure 5 shows the LabVIEW program. 
Paper-Smart Sensor Network Based High Quality Air Pollution Monitoring System Using Labview

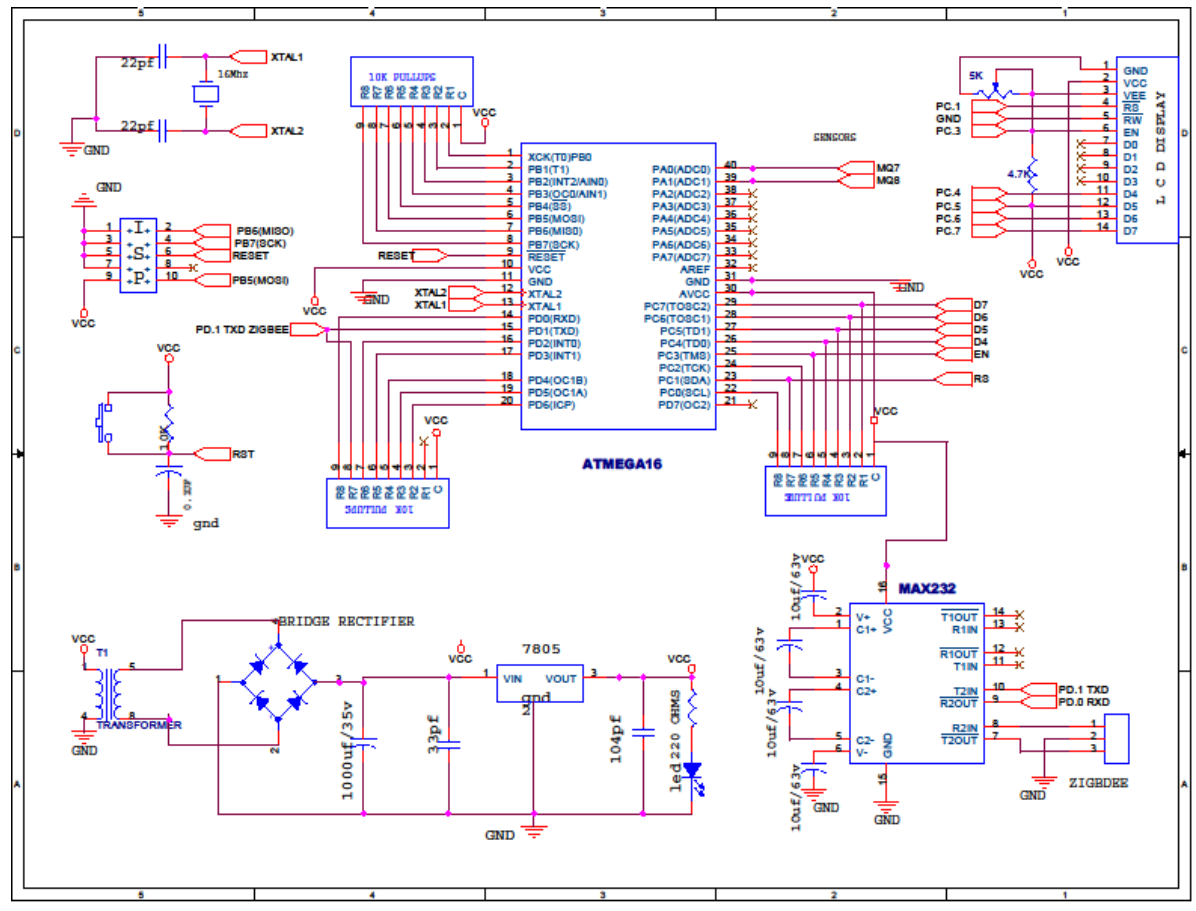

Fig. 4. Schematic diagram of Proposed Architecture

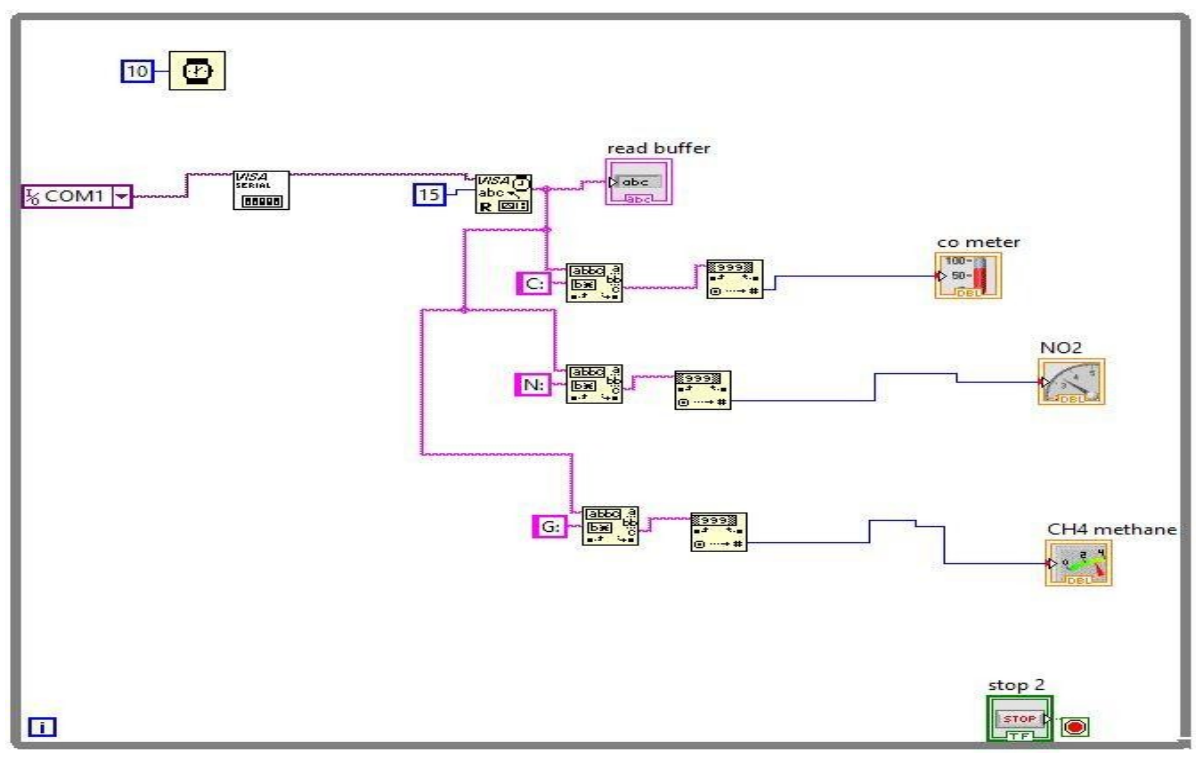

Fig. 5. Graphical programming model for Air pollution monitoring system 


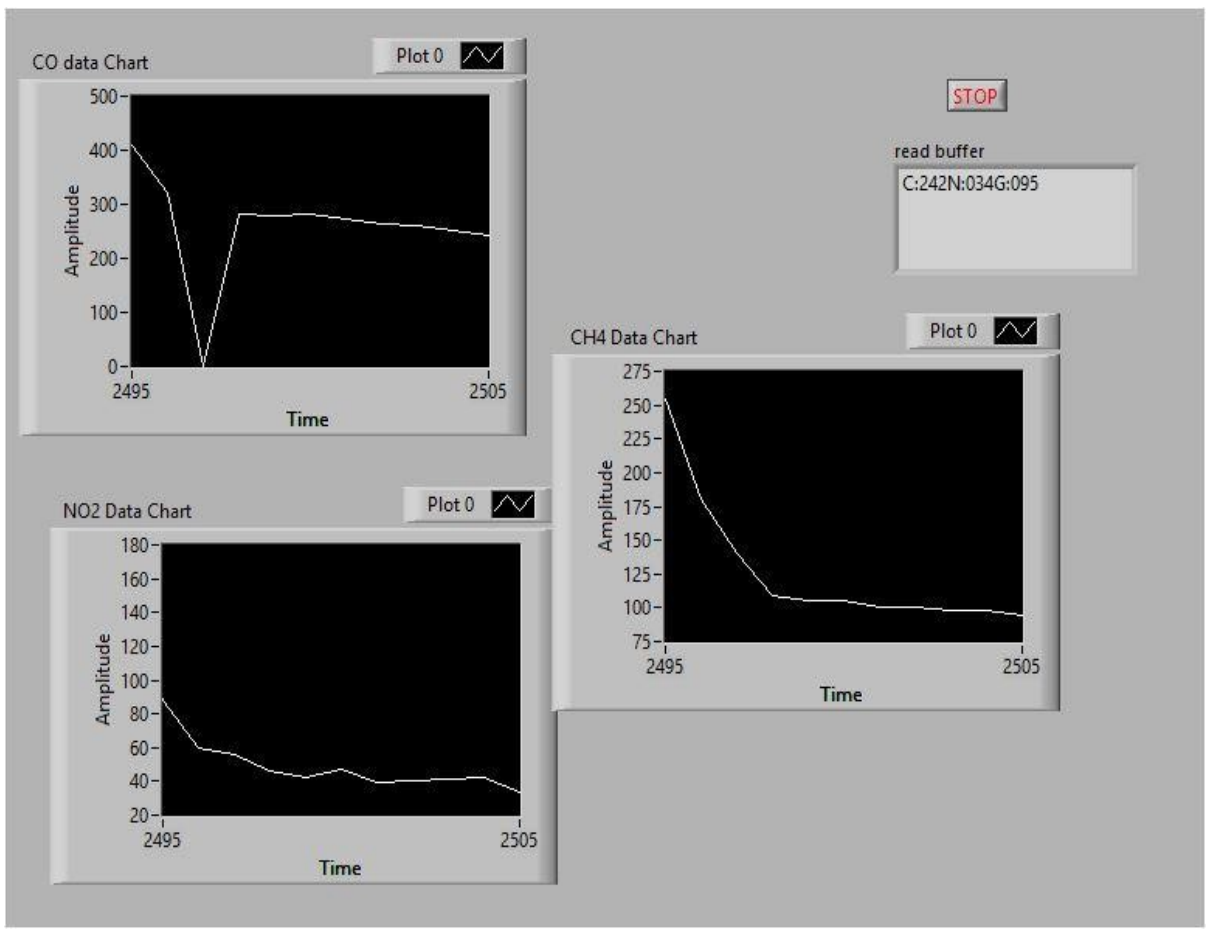

Fig. 6. Front panel output showing Graphs of MQ-7, MQ-135 Sensors output

The waveform chart maintains a history of data from previous updates. The read buffer contains the serial data which is of 15 Bytes of data. In this figure the charts are plotted from the output of string to numeric function. The charts specify the amplitude on $\mathrm{Y}$-axis and time on $\mathrm{X}$-axis. Here the different air pollutants such as Nitrous oxide, carbon mono oxide and sulphorous oxide analog data is measured in the embedded system kit through microprocessor and then data is given to Zigbee module such that with the help of VISA driver installed in the laptop/computer i.e it acts as serial communication port with $\mathrm{I} / \mathrm{O}$ comm. port the data is plotted with chart present on the front panel of the LabVIEW. For the different timing the chart specifies different amplitudes, from the charts we can analyze the data and interpret the mostly affected area in the city. Figure 10 shows the charts used in the front panel. 


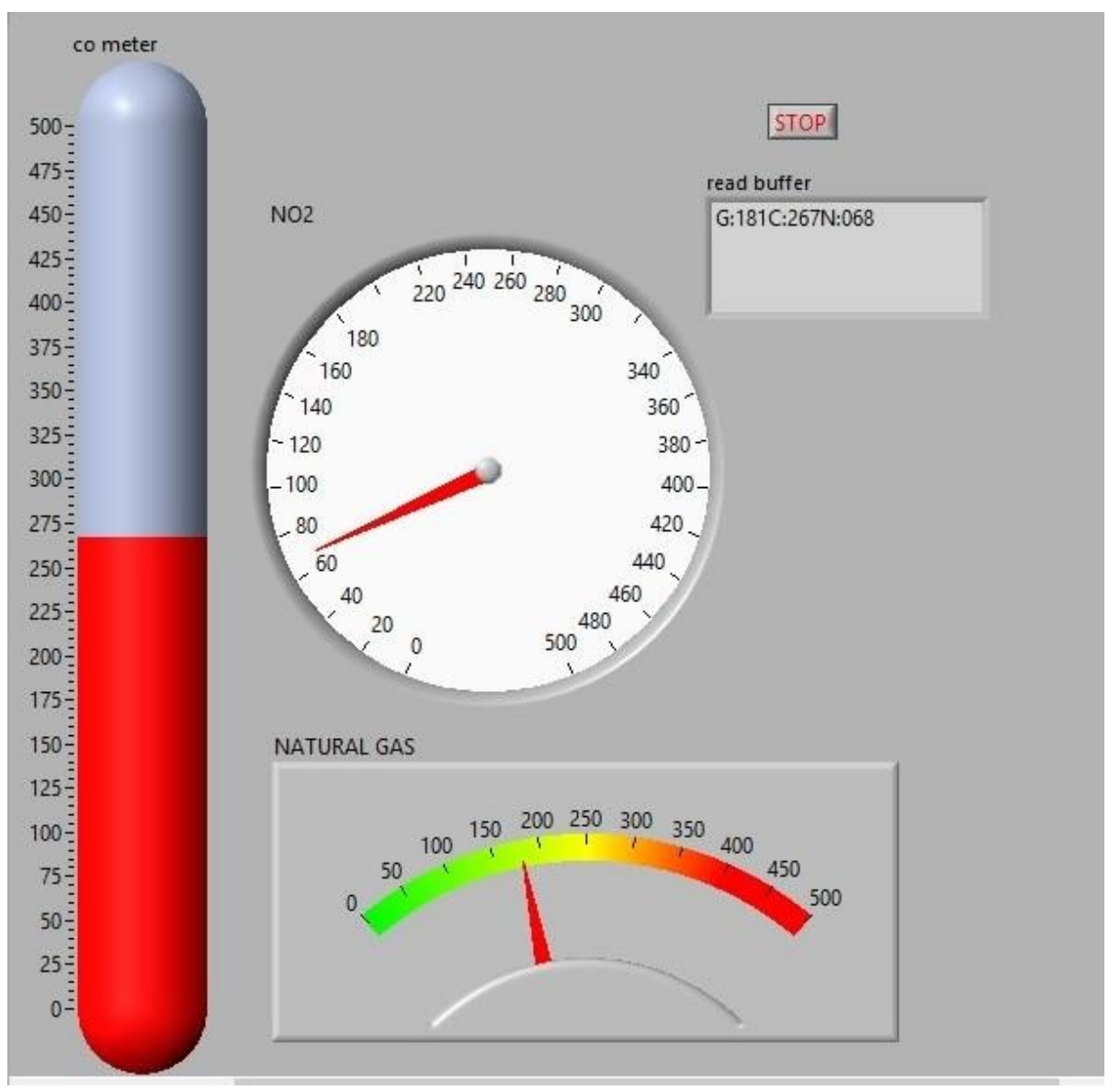

Fig. 7. Front panel showing indicators of sensors output and read buffer output

Use numeric controls and indicators on the front panel to enter and display numeric data in LabVIEW applications. Here three different indicators are used for measurement of different air pollutants. The first one is temperature indicator in which the display format is numeric in SI notation. It indicates the amount of carbon monoxide content in the atmosphere. Another indicator we used is screw gauge. The needle color is red which indicator the amount of nitrous oxide in the atmosphere. The last indicator we used is meter which indicates the content of natural gas in the atmosphere. Figure 11 shows the the front panel for different sensor data.

\section{Conclusion}

The major issue now-a-days is regarding the pollution of air. After few decades, there may not be pollution free area on the earth. So there is an urgency to check the 
pollution in populated areas through which measurement of harmful gases can be done.

A low-cost low complexity and scalable wireless mash network based real-time air quality monitoring system is presented which includes carbon monoxide, nitrogen dioxide and methane gas measurement. By constructing the above model, we can take measures regarding the pollution control in the public areas and pave way for controlling pollution.

\section{References}

[1] S.N.Talukdar, R. D. Brook, B. Franklin, W. Cascio, Y. Hong, G. Howard, M Lipsett, R. Luepker, M. Mittleman, J. Samet, S. C. Smith Jr, I. Tager.(2004). Air pollution and cardiovascular disease: A statement for healthcare professionals from the Expert Panel on Population and Prevention Science of the American Heart Association, Circulation, 109(21), 2655-2671. https://doi.org/10.1161/01.CIR.0000128587.30041.C8

[2] Book, UNEP Year.(2014). Emerging issues update Air Pollution: World's Worst Environmental Health Risk. United Nations Environment Programme,

[3] World Health Organization.(2014).Ambient (outdoor) air quality and health.Fact sheet313.

[4] N. A. Clark, P. A. Demers, C. J. Karr, M. Koehoorn, C. Lencar, L. Tamburic, M. Brauer.(2010). Effect of early life exposure to air pollution on development of childhood asthma,118(2), 284-290.

[5] R. McConnell R, T. Islam, K. Shankardass, M. Jerrett, F. Lurmann, F. Gilliland, J. Gauderman, E. Avol, N. Künzli, L. Yao, J. Peters, K. Berhane.(2010). Childhood incident asthma and traffic-related air pollution at home and school, 118(7), 1021-2026.

[6] C. Carlsten, A. Dybuncio, A. Becker, M. Chan-Yeung, M. Brauer. (2011). Trafficrelated air pollutionand incident asthma in a high-risk birth cohort," 68(4), 291-295.

[7] J. K. Mann, J. R. Balmes, T. A. Bruckner, K. M. Mortimer, H. G. Margolis, B. Pratt, S. K. Hammond, F. W. Lurmann, I. B. Tager.(2010). Short-term effects of air pollution on wheeze in asthmatic children in Fresno, 118(10), 1497-1502.

[8] P. Mohai, B.-S.Kweon, S. Lee, K. Ard.(2011). Air pollution around schools is linked to poorer studenthealth and academic performance, 30(5), 852-862.

[9] Ali, H., J. K. Soe, Steven R. Weller.(2015). A real-time ambient air quality monitoring wireless sensor network for schools in smart cities. Smart CitiesConference (ISC2), IEEE FirstInternational. https://doi.org/10.1109/ISC2.2015.7366163

[10] W. J. Gauderman, E. Avol, F. Gilliland, H. Vora, D. Thomas, K. Berhane, R. McConnell, N. Kuenzli, F. Lurmann, E. Rappaport, H. Margolis, D. Bates, J.Peters.(2004). The effect of air pollution on lung development from 10 to 18 years of age, 351(11), 1057-1067

\section{Authors}

Nagarjuna Telagam (corresponding author) is with the Electronics and Communication Engineering Department, Institute of Aeronautical Engineering, Hyderabad, India. Currently he is Ph.D Research Scholar in Sathyabama University, Chennai and is interested in the following topics: Wireless Communications, MIMO, OFDM, 
GFDM. Currently, He is working as Assistant Professor. He received his Bachelor degree from JNTU University/ Narayana Engineering College in 2011. He received his Master degree from Anna University/ Loyola Institute of Technology in 2013. He is Anna University rank holder(20) for M.E degree in2013.He has nearly 4 years of experience in teaching and research. He published papers in different referred Journals (nagarjuna473@gmail.com).

Nehru Kandasamy received his Bachelor Degree in Erode Sengunthar Engineering College, Anna University in 2005. And he obtained his Master Degree in R.M.K Engineering, Anna University in 2007. And he also obtained outstanding master student at that year. He is obtained his Ph.D in 2014 at Faculty of Information and Communication Engineering, Anna University, India. His main research interest is in the area of Low Power VLSI, Testing of VLSI Circuits, FPGA Design, CAD for VLSI, Signal processing. He has published papers on these topics in various international journals.(nnehruk@gmail.com).

Nagendra Prasad G is with the Electronics and Communication Engineering Department, Institute of Aeronautical Engineering, Hyderabad, India. He received his Bachelor degree from Sri Venkateswara University in 2002. He received his Master degree from Sri Venkateswara University in 2006. Currently he is working as Associate Professor. His professional experience is about 9 years in teaching. His areas of interest are Electromagentic Field theory and transmission lines, Antenna wave propagation and microwave Engineering. He has published papers on these topics in various international journals. (nagendraprasadg@gmail.com)

Menakadevi Nanjundan is with the Electronics and Communication Engineering Department, Hindustan College of Engineering and Technology, Coimbatore, India. She is a Research Fellow and is interested in the following topics: Digital Image processing, Wireless communications. Currently She is working as assistant professor. Her professional experience is about 5years in the field of teaching. She completed Master degree M.E - communication system in Loyola institute of technology/Anna University Chennai in the year 2013. She is Anna University Rank holder (22) for her M.E degree. She finished her Bachelor degree B.E - electronics and communication engineering in muthayammal engineering college in 2006. She published various papers in different referred journals. (menakadevin@gmail.com)

Article submitted 13 May 2017. Published as resubmitted by the authors 23 June 2017. 